\title{
Dank an die Gutachter des Jahres 2021
}

Bei der Begutachtung und Durchsicht von Manuskripten für die RöFo haben uns im vergangenen Jahr zahlreiche Experten in Kliniken und Instituten unterstützt, die nachfolgend genannt sind und denen die Herausgeber der RöFo und der Verlag für ihre Hilfe herzlich danken.

Zunächst genannt seien die Gutachter, die für ihr besonderes Engagement ausgezeichnet wurden:

\author{
Mark Wielpütz, Heidelberg \\ Marc-André Weber, Rostock \\ Martin Maurer, Bern \\ Andreas Schreyer, Brandenburg/Havel \\ Dag Wormanns, Berlin \\ Marco Das, Duisburg \\ Frank Oliver Henes, Hamburg
}

Gerhard Adam, Hamburg, Deutschland Saif Afat, Tübingen, Deutschland Thomas Albrecht, Berlin, Deutschland Hatem Alkadhi, Zurich, Schweiz Christoph Artzner, Tübingen, Deutschland Ingolf Askevold, Gießen, Deutschland Pascal Andreas Thomas Baltzer, Wien, Österreich

Fabian Bamberg, Freiburg, Deutschland Ralf W. Bauer, Wiesbaden, Deutschland Thomas Baum, München, Deutschland Alexander Baur, Berlin, Deutschland Meinrad Beer, Ulm, Deutschland Dietrich Beitzke, Wien, Österreich Benjamin Bender, Tübingen, Deutschland Ansgar Berlis, Augsburg, Deutschland Dirk Beyersdorff, Hamburg, Deutschland Jürgen Biederer, Heidelberg, Deutschland Christoph Binkert, Winterthur, Schweiz Annalen Bleckmann, Münster, Deutschland Thorsten Bley, Würzburg, Deutschland Karin Bock, Marburg, Deutschland Markus Born, Bonn, Deutschland Markus Borowski, Braunschweig, Deutschland

Marcus Both, Kiel, Deutschland Andreas Bucher, Frankfurt, Deutschland Johannes Budjan, Mannheim, Deutschland Alexander Christian Bunck, Köln,

Deutschland

Arno Bücker, Homburg, Deutschland Svenja Caspers, Düsseldorf, Deutschland Marco Das, Aachen, Deutschland Thomas Decker, Neubrandenburg, Deutschland

Lena Marie Dendl, Treuenbrietzen, Deutschland

Timm Denecke, Leipzig, Deutschland
Marc Dewey, Berlin, Deutschland Stefan Diederich, Düsseldorf, Deutschland Olaf Dietrich, München, Deutschland Matthias Dietzel, Jena, Deutschland Matthias Dietzel, Erlangen, Deutschland Felix Doellinger, Berlin, Deutschland Anja Eckstein, Essen, Deutschland Constantin Ehrengut, Leipzig, Deutschland Tobias Engelhorn, Erlangen, Deutschland Kathrin Entz, Frankfurt am Main, Deutschland

Arne Estler, Tübingen, Deutschland Cornelius Faber, Münster, Deutschland Anton Faron, Bonn, Deutschland Jens Fiehler, Hamburg, Deutschland Lutz Fischer, Hamburg, Deutschland Jochen Fleckenstein, Homburg, Deutschland Cornelia Focke, Neubrandenburg, Deutschland

Borek Foldyna, Boston, MA, USA Michael Forsting, Essen, Deutschland Tobias Franiel, Jena, Deutschland Bernd Frericks, Berlin, Deutschland Peter Fries, Homburg, Deutschland Jan Fritz, New York, USA Marco Galster, Nürnberg, Deutschland Sebastian Gassenmaier, Tübingen, Deutschland Bernhard Gebauer, Berlin, Deutschland Dominik Geisel, Berlin, Deutschland Joachim Gerss, Münster, Deutschland Lucas L. Geyer, München, Deutschland Elke R. Gizewski, Innsbruck, Österreich Susanne Grandl, München, Deutschland Thoas Grieser, Augsburg, Deutschland Johannes, Stefan, Grueneisen, Essen, Deutschland

Andreas Gröschel, Münster, Deutschland
Gerd Grözinger, Tübingen, Deutschland Matthias Gutberlet, Leipzig, Deutschland Friedrich Götz, Hannover, Deutschland Rolf Wilhelm Günther, Berlin, Deutschland Christian R. Habermann, Hamburg, Deutschland Peter Hallscheidt, Heidelberg, Deutschland Okka Wilkea Hamer, Regensburg,

Deutschland

René Hartensuer, Münster, Deutschland Elke Hattingen, Frankfurt, Deutschland Sabine Heiland, Heidelberg, Deutschland Walter Heindel, Münster, Deutschland Peter Heiss, Regensburg, Deutschland Karin Hellerhoff, München, Deutschland Thomas Helmberger, München, Deutschland Frank Oliver Gerhard, Henes, Hamburg, Deutschland Jan Philipp Hering, Ibbernburen, Deutschland Jochen Herrmann, Hamburg, Deutschland Ken Herrmann, Essen, Deutschland Christoph M. Heyer, Bochum, Deutschland Sylvia, Helen, Heywang-Köbrunner, München, Deutschland Jan B. Hinrichs, Hannover, Deutschland Wolfgang Hirsch, Leipzig, Deutschland Karl-Titus Hoffmann, Leipzig, Deutschland Joachim Hohmann, Basel, Schweiz Marius Horger, Tübingen, Deutschland Norbert Hosten, Greifswald, Deutschland Peter Hunold, Göttingen, Deutschland Martin Härter, Hamburg, Deutschland Peter Isfort, Aachen, Deutschland Werner Jaschke, Innsbruck, Österreich Kai Uwe Juergens, Bremen, Deutschland Ernst Michael Jung, Regensburg, Deutschland Jörg Jüngert, Erlangen, Deutschland Clemens Kaiser, Mannheim, Deutschland 
Manoj Kakkassery, Berlin, Deutschland Marian Kampschulte, Gießen, Deutschland Marcus Katoh, Krefeld, Deutschland Hans-Ulrich Kauczor, Heidelberg, Deutschland

Julian Kirchner, Düsseldorf, Deutschland Stephan Klebe, Essen, Deutschland Christopher Kloth, Ulm, Deutschland Ulrich Kramer, Winnenden, Deutschland K.-F. Kreitner, Mainz, Deutschland Ilonka Kreitschmann-Andermahr, Essen, Deutschland

Christian R. Krestan, Wien, Österreich Gabriele Anja Krombach, Gießen, Deutschland Patrick Krumm, Tübingen, Deutschland Tobias Krähling, Münster, Deutschland Michael Köhler, Münster, Deutschland Herbert Köstler, Würzburg, Deutschland Mark E. Ladd, Heidelberg, Deutschland Heinrich Lanfermann, Hannover, Deutschland

Tim Leiner, Utrecht, Niederlande Simon Lennartz, Köln, Deutschland Markus Herbert, Lerchbaumer, Berlin, Deutschland

Sebastian Ley, München, Deutschland Mario Liebmann, Bremen, Deutschland Christoph G. Lisson, Ulm, Deutschland André Lollert, Mainz, Deutschland Joachim Lotz, Göttingen, Deutschland Martin Lotze, Greifswald, Deutschland Andreas H. Mahnken, Marburg, Deutschland Marcus R. Makowski, Berlin, Deutschland Milka Marinova, Bonn, Deutschland Simon S. Martin, Frankfurt, Deutschland Alexander Massmann, Homburg,

Deutschland

Martin H. Maurer, Bern, Schweiz Matthias Stefan May, Erlangen, Deutschland Marius Mayerhoefer, Wien, Österreich Mathias Meyer, Hamburg, Deutschland Peter Mildenberger, Mainz, Deutschland Peter Minko, Homburg/Saar, Deutschland Walter Moeller-Hartmann, Meppen, Deutschland

Nuschin Morakkabati-Spitz, Bonn, Deutschland

Ullrich Gerd Mueller-Lisse, München, Deutschland

Christoph Mönninghoff, Minden, Deutschland Jonathan Nadjiri, München, Deutschland Kai Nassenstein, Essen, Deutschland Henning Neubauer, Suhl, Deutschland
Claus Nolte-Ernsting, Mülheim an der Ruhr, Deutschland

Claas Philip Nähle, Bonn, Deutschland

Jörg Oestmann, Berlin, Deutschland

Daniel Paech, Bonn, Deutschland

Panagiotis Papanagiotou, Bremen, Deutschland

Philipp M. Paprottka, München, Deutschland Maciej Pech, Magdeburg, Deutschland Lenhard Pennig, Köln, Deutschland Bernhard Petritsch, Würzbug, Deutschland Claus Christian Pieper, Bonn, Deutschland Daniel Pinto dos Santos, Koln, Deutschland Heike Preibsch, Tübingen, Deutschland Helmut Prosch, Wien, Österreich Michael Puesken, Köln, Deutschland Paul Raczeck, Homburg, Deutschland Rolf Reiter, Berlin, Deutschland Bernhard Renger, München, Deutschland Diane Miriam Renz, Hannover, Deutschland Christoph Riedel, Hamburg, Deutschland Philipp Riffel, Mannheim, Deutschland Helmut Ringl, Wien, Österreich Christian O. Ritter, Göttingen, Deutschland Robert M. Ritzel, Hamburg, Deutschland Julian Rogasch, Homburg, Deutschland Fritz Christian Roller, Gießen, Deutschland Bastian O. Sabel, München, Deutschland Maliha Sadick, Mannheim, Deutschland Johannes Salamon, Hamburg, Deutschland Alexander Sauter, Basel, Schweiz Thomas Sauter, München, Deutschland Michael Schaefers, Münster, Deutschland Rüdiger Schernthaner, Wien, Österreich Christian Scheurig-Münkler, Augsburg, Deutschland

Fritz Schick, Tübingen, Deutschland Lars Schimmöller, Duesseldorf, Deutschland Kathrin Schindler, Erfurt, Deutschland Marc Schlamann, Köln, Deutschland Heinz-Peter Schlemmer, Heidelberg, Deutschland Thomas Schlosser, Essen, Deutschland Jörg Schmehl, Tübingen, Deutschland Bernd Ludwig Schmitz, Ulm, Deutschland

U. Joseph Schoepf, Charleston, USA

Stephan Schopphoven, Marburg, Deutschland

Andreas G. Schreyer, Brandenburg a.d. Havel, Deutschland

Klaus Schunk, Koblenz, Deutschland Hannes Schwenke, Lubeck, Deutschland Stefan O. Schönberg, Mannheim, Deutschland

Christoph Schülke, Münster, Deutschland
Max Seidensticker, München, Deutschland Stephan Skornitzke, Heidelberg, Deutschland Gregor Sommer, Basel, Schweiz Alexander Sommer, Münster, Deutschland Ina Sorge, Leipzig, Deutschland Clemens Spink, Hamburg, Deutschland Fabian Springer, Tübingen, Deutschland Gundula Staatz, Mainz, Deutschland Georg Stamm, Hannover, Deutschland Marc Steinborn, München, Deutschland Corinna Storz, Tübingen, Deutschland Florian Streitparth, München, Deutschland Christian Stroszczynski, Regensburg, Deutschland

Enver Tahir, Hamburg, Deutschland Matthias Taupitz, Berlin, Deutschland Ulf Teichgräber, Jena, Deutschland Wolfgang Maximilian Thaiss, Tübingen, Deutschland

Jens M. Theysohn, Essen, Deutschland Siegfried Trattnig, Wien, Österreich Christoph Gregor Trumm, München, Deutschland

Bernd Turowski, Düsseldorf, Deutschland Wibke Uller, Freiburg, Deutschland Tim Ullrich, Düsseldorf, Deutschland Jens Vogel-Claussen, Hannover, Deutschland Thomas J. Vogl, Frankfurt, Deutschland Dierk Vorwerk, Ingolstadt, Deutschland Frank K. Wacker, Hannover, Deutschland Michael Walz, Eschborn, Deutschland Mike Watjes, Hannover, Deutschland Christoph Weber, Hamburg, Deutschland Marc-André Weber, Rostock, Deutschland Werner Weber, Bochum, Deutschland Julius Matthias Weinrich, Hamburg, Deutschland

Christina, M., Wendl, Regensburg, Deutschland

Andreas Wenke, Münster, Deutschland Rene Werner, Hamburg, Deutschland Johannes Wessling, Münster, Deutschland Axel Wetter, Essen, Deutschland C. Wiedenroth, Bad Nauheim, Deutschland Mark Oliver Wielpütz, Heidelberg, Deutschland

Benedikt Wiestler, München, Deutschland Moritz Wildgruber, München, Deutschland Bernd Wintersperger, Toronto, Kanada Florian Wolf, Wien, Österreich Dag Wormanns, Berlin, Deutschland Dominik Zinsser, Tübingen, Deutschland Tarek Zoubi, Ibbenbüren, Deutschland 